\title{
Sarcoidosis in a time of pandemic
}

\author{
Praveen Govender ${ }^{1,2}$ and Yvette C. Cozier ${ }^{3,4}$
}

Affiliations: ${ }^{1}$ Dept of Pulmonary, Allergy, Sleep and Critical Care Medicine, Boston University School of Medicine, Boston, MA, USA. ${ }^{2}$ The Sarcoidosis Clinic at Boston Medical Center, Boston, MA, USA. ${ }^{3}$ Slone Epidemiology Center at Boston University, Boston University School of Medicine, Boston, MA, USA. ${ }^{4}$ Dept of Epidemiology, Boston University School of Public Health, Boston, MA, USA.

Correspondence: Yvette C. Cozier, Slone Epidemiology Center at Boston University, 72 East Concord Street, Boston, MA 02118, USA. E-mail: yvettecRabu.edu

@ERSpublications

Sarcoidosis patients treated with immunosuppressants have a higher risk of serious, recurrent infection. The high morbidity and mortality associated with COVID-19 require a cautious re-evaluation of short- and long-term sarcoidosis treatments. https://bit.ly/2YwZ9wb

Cite this article as: Govender P, Cozier YC. Sarcoidosis in a time of pandemic. Eur Respir J 2020; 56: 2002376 [https://doi.org/10.1183/13993003.02376-2020].

We are living in a historic event: a novel virus, severe acute respiratory syndrome coronavirus 2 (SARS-CoV-2), emerged in Wuhan, China at the end of 2019 and began its global circulation. By January 24, 2020, at least 830 cases and 26 fatalities were reported across Asia and the USA [1]. 6 months later, there are more than 8 million confirmed cases of the disease associated with SARS-CoV-2, coronavirus disease 2019 (COVID-19), and over 430000 reported deaths worldwide [2]. The pandemic has exposed cracks in healthcare systems throughout the world and identified populations vulnerable for severe disease and poorer outcomes, including the elderly, "essential workers" of lower socioeconomic class, and residents of nursing homes [3]. The Centers for Disease Control (CDC) also state that those with chronic lung disease, namely asthma and COPD, the obese, and those with "prolonged use of corticosteroids and other immune weakening medications" might be at increased risk for COVID-19 related illness [3].

Although not specifically named in the CDC report, the list of underlying conditions accurately describe many patients with sarcoidosis, a systemic granulomatous disease that frequently affects the lung. It should not be surprising, in this pandemic, that these patients, and their physicians, are anxiously wondering whether they are more susceptible to COVID-19 or whether they might have a more severe clinical course. Thus, the report from Rossides et al. [4] in the current issue of the European Respiratory Journal, is timely. While not explicitly answering the question of susceptibility of sarcoidosis patients to COVID-19 infection, the paper addresses this important concern in general and highlights an under-researched area in sarcoidosis literature: risk of secondary infections.

In their analysis, Rossides et al. [4] examined hospitalisations and outpatient visits recorded in the Swedish National Patient Register to determine risk of serious hospitalised infections (SI), defined as a hospitalisation with an infectious disease as the primary discharge code (ICD-9). Over 8700 newly diagnosed sarcoidosis cases identified from 2003-2013 were matched to 86000 general population controls on age, sex and residential location. Information on initial immunosuppressive therapy was available for $77 \%$ of the study population. They found that rates of SI and recurrent SI were 1.8-fold and 2.8-fold higher, respectively, in patients with sarcoidosis compared to the general population.

The relationship of sarcoidosis with infection is complex. Sarcoidosis is an exuberant or inappropriate granulomatous response to an undefined antigen(s) [5]. Among the many triggers known to cause granulomas, infection is the most common [6]. Additionally, there are well-known infectious syndromes 
that complicate sarcoidosis (e.g. chronic pulmonary aspergillosis) [7], but the incidence of this and other infectious complications is low [7, 8]. For example, WinTERBAUER and Kraemer [9] reported opportunistic infections in five of $122(4.1 \%)$ sarcoidosis patients in Seattle, WA, USA over a 7-year follow-up period. Further, there was no increased incidence of infection in comparison to normal controls, despite $71 \%$ of the patients having pulmonary involvement and $39 \%$ being on steroids. A retrospective record review by BAUGHMAN and LOWER [8] of clinic-based sarcoidosis patients found that only 7 out of $753(0.9 \%)$ developed fungal infections over an 18-month period. In 2017, a hospital-based, retrospective cohort study of 585 French sarcoidosis patients, estimated a 5\% frequency of severe infections (i.e. resulting in hospitalisation or death) over an 8-year (median) follow-up [10]. In accordance with the findings of Winterbauer and Kraemer [9], the low frequency of infection observed in the latter two studies was in spite of high usage of corticosteroid therapy: $100 \%$ [8] and $82 \%$ [10], respectively.

Conversely, results from a retrospective case-cohort study in Olmstead County, MN, USA from 1976 to 2013, suggested a diagnosis of sarcoidosis conferred a higher risk of infection requiring hospitalisation (hazard ratio (HR) 2.00, 95\% CI 1.33-6.90). Use of steroids was a significant predictor of hospitalised infection; the hazard ratios for daily dosages of $\leqslant 10 \mathrm{mg}$ and $>10 \mathrm{mg}$ were 3.03 and 4.48 , respectively [11].

The current paper by Rossides et al. [4] re-emphasises this latter observation; the negative impact of therapy. Immunosuppressive therapy is recommended, according to Wells's Law [12], for impaired quality of life or fear of danger (i.e. threatened organ failure). Corticosteroids are considered first line of treatment due to their ability to rapidly suppress granulomatous inflammation [13]. Patients can relapse upon withdrawal, thus these agents are often prescribed for prolonged periods resulting in side-effects that can significantly impact quality of life [14]. In the current analysis, risk of first SI was three-fold higher among those treated with immunosuppressants compared to those who were not [4]. Although the indications for therapy, the length of therapy, and the cumulative dose of immunosuppressants used in the sarcoidosis cohort are unknown, prescribed treatment alone at the start of diagnosis was sufficient to increase one's risk for infection.

As the authors acknowledge, several study methods utilised impact the accuracy and validity of their results. These include the reliance on ICD coding to define both the study population and the outcome diagnosis, as well as use of prescriptions as surrogate for immunosuppressant use. Sarcoidosis encompasses heterogeneous clinical phenotypes and even patients with similar presentations have varied clinical courses. The paper does not address whether sarcoidosis in general or different phenotypes of sarcoidosis (e.g. Löfgren syndrome, non-Löfgren syndrome) result in worse clinical outcomes following hospitalisation. Equally, it does not address whether the identified increased risk of SI affects quality of life. A survey conducted by the European Lung Foundation Sarcoidosis Patient Advisory Group demonstrated that patients view quality of life as an important aspect of treatment that should be assessed and incorporated into clinical care [15]. It is clear that further research in this area is needed.

So, should sarcoidosis patients be worried about SI? Yes and no. On the one hand, patients can be reassured that opportunistic infection is infrequent despite high corticosteroid use. This is shown in previous studies [8-10], and in the current study data, where after adjustment for having one or more healthcare visits in the 2 years prior to diagnosis, the risk of SI in untreated, newly diagnosed patients sarcoidosis appeared to be minimal (adjusted HR 1.03, 95\% CI 0.88-1.22) [4]. On the other hand, risk for serious infections (i.e. hospitaliseable infection) is higher in treated, newly diagnosed patients [4].

Should sarcoidosis patients be worried about COVID-19 specifically? This is harder to answer. Approximately 2\% of patients with COVID-19 have simultaneous pulmonary disease and experience worse outcomes than those without [16]. Given the high morbidity and mortality associated with COVID-19 infection, there is concern that the risk-benefit calculation regarding immunosuppressive therapies for sarcoidosis may become unfavourable [17]: continued treatment might increase risk of infection while therapeutic taper may increase symptoms. Despite this concern, it has yet to be established whether sarcoidosis is a risk for development of disease or for poorer outcomes.

In light of the COVID-19 pandemic, SwEISs et al. [17] have issued an opinion statement stressing the importance of minimising the initial treatment dose of immunosuppression therapy, which is the standard, accepted practice in the management of sarcoidosis. They further call upon clinicians to taper existing patients to the lowest effective dose [17]. While this may appear straightforward, disparity in socioeconomic status may make such adjustment difficult to achieve.

A recent paper from the USA by HARPER et al. [18] found an inverse association between socioeconomic status and long-term, steroid-related comorbid conditions among sarcoidosis patients. Lower socioeconomic status individuals in the USA may have limited ability to take time off from work for follow-up clinic visits, and additional costs associated with physician visits (e.g. transportation, insurance 
co-payments) may make multiple clinic visits burdensome [19]. However, Sweden has a tax-funded, universally accessible healthcare system, therefore, it is not clear from the current analysis whether Swedish patients face similar impediments to care [4]. Additionally, Sweden and the USA differ in terms of racial composition, healthcare access, and the distribution of chronic health conditions (e.g. obesity, type-2 diabetes) [20-22].

ROssides et al. [4] have helped us to understand generally the risk of serious hospitalised infections in newly diagnosed cases of sarcoidosis, and highlights that the initial decision to treat is important as it could have lasting harmful effects. The COVID-19 pandemic has exposed major social and financial fissures globally. Furthermore, recent events in the USA have amplified these fault lines. Racial and social disparities undergird much of contemporary American society, and to a different extent, societies around the globe. Since sarcoidosis disproportionately affects the lives of persons with low socioeconomic status and racial minorities (for example, African Americans/Blacks), the article by Rossides et al. [4] also serves as a reminder that sarcoidosis straddles the historic convergence of the COVID-19 pandemic and exposure of societal inequality in healthcare outcomes.

Acknowledgement: We are grateful to Jeffrey Berman for his editorial feedback and mentorship.

Conflict of interest: None declared.

Support statement: Y.C. Cozier is supported by the National Cancer Institute U01-CA164974, and the National Institute of Arthritis and Musculoskeletal and Skin Diseases R01 AR0573727. Funding information for this article has been deposited with the Crossref Funder Registry.

\section{References}

1 Munster VJ, Koopmans M, van Doremalen N, et al. A novel coronavirus emerging in china - key questions for impact assessment. N Engl J Med 2020; 382: 692-694.

2 Johns Hopkins University. COVID-19 Map. 2020. https://coronavirus.jhu.edu/map.html Date last accessed: 12 June 2020.

3 Centers for Disease Control and Prevention. Coronavirus Disease 2019 (COVID-19). Centers for Disease Control and Prevention. 2020. www.cdc.gov/coronavirus/2019-ncov/need-extra-precautions/racial-ethnicminorities.html Date last accessed: 12 June 2020.

4 Rossides M, Kullberg S, Eklund A, et al. Risk of first and recurrent serious infection in sarcoidosis: a Swedish register-based cohort study. Eur Respir J 2020; 56: 2000767.

$5 \quad$ Newman LS, Rose CS, Maier LA. Sarcoidosis. N Engl J Med 1997; 336: 1224-1234.

6 Zumla A, James DG. Granulomatous infections: etiology and classification. Clin Infect Dis 1996; 23: 146-158.

7 Denning DW, Pleuvry A, Cole DC. Global burden of chronic pulmonary aspergillosis complicating sarcoidosis. Eur Respir J 2013; 41: 621-626.

8 Baughman RP, Lower EE. Fungal infections as a complication of therapy for sarcoidosis. QJM 2005; 98: 451-456.

9 Winterbauer RH, Kraemer KG. The infectious complications of sarcoidosis: a current perspective. Arch Intern Med 1976; 136: 1356-1362.

10 Dureault A, Chapelon C, Biard L, et al. Severe infections in sarcoidosis: incidence, predictors and long-term outcome in a cohort of 585 patients. Medicine (Baltimore) 2017; 96: e8846.

11 Ungprasert P, Crowson CS, Matteson EL. Sarcoidosis increases risk of hospitalized infection. A population-based study, 1976-2013. Ann Am Thorac Soc 2017; 14: 676-681.

12 Baughman RP, Judson MA, Wells A. The indications for the treatment of sarcoidosis: Wells Law. Sarcoidosis Vasc Diffuse Lung Dis 2017; 34: 280-282.

13 Gibson GJ, Prescott RJ, Muers MF, et al. British Thoracic Society Sarcoidosis study: effects of long term corticosteroid treatment. Thorax 1996; 51: 238-247.

14 Judson MA, Chaudhry H, Louis A, et al. The effect of corticosteroids on quality of life in a sarcoidosis clinic: the results of a propensity analysis. Respir Med 2015; 109: 526-531.

15 Baughman RP, Barriuso R, Beyer K, et al. Sarcoidosis: patient treatment priorities. ERJ Open Res 2018; 4: 00141-2018.

16 Yang J, Zheng Y, Gou X, et al. Prevalence of comorbidities and its effects in patients infected with SARS-CoV-2: a systematic review and meta-analysis. Int J Infect Dis 2020; 94: 91-95.

17 Sweiss NJ, Korsten P, Syed H, et al. When the game changes: guidance to adjust sarcoidosis management during the COVID-19 pandemic. Chest 2020; in press [https://doi.org/10.1016/j.chest.2020.04.033].

18 Harper L, Gerke AK, Wang X, et al. Income and other contributors to poor outcomes in US sarcoidosis patients. AJRCCM. Am J Respir Crit Care Med 2020; 201: 955-964.

19 Himmelstein DU, Lawless RM, Thorne D, et al. Medical bankruptcy: still common despite the affordable care act. Am J Public Health 2019; 109: 431-433.

20 Statistics Sweden. www.scb.se/en/finding-statistics/ Date last accessed: 15 June 2020.

21 US Census Bureau. www.census.gov/topics/health.html Date last accessed: 17 June 2020.

22 National Center for Health Statistics. www.cdc.gov/nchs/ Date last accessed: 17 June 2020 